\title{
Aprendizaje basado en congresos. XII Congreso de Educación Médica en la Universidad de Alcalá
}

\author{
Celia Estrada Costas 1, *, Paula Rubio García ${ }^{2, *}$ \\ 1 Estudiante de Medicina (promoción 2013-2019); Universidad de Alcalá; \\ 2 Estudiante de Medicina (promoción 2013-2019); Universidad de Alcalá; \\ * Autor correspondencia: presidenciacoxiem@gmail.com
}

Recibido: 28/05/2019; Aceptado: 30/05/2019; Publicado: 31/05/2019

Resumen: El XII Congreso de Educación Médica organizado por el Consejo Estatal de Estudiantes de Medicina (CEEM) con sede en Universidad de Alcalá es el encuentro anual más importante para el estudiantado de Medicina a nivel nacional. Este evento pretende ampliar la formación en aspectos científicos y sociales de los futuros profesionales de la salud. En este estudio se analiza la utilidad y calidad del aprendizaje basado en congresos mediante dos encuestas enviadas al estudiantado de medicina antes y después del congreso. Para el análisis estadístico se utilizó un test de diferencias porcentuales. Nuestros resultados muestran la eficacia del Congreso como herramienta innovadora de aprendizaje en el Grado en Medicina. Por esto se considera adecuado seguir fomentando las actividades organizadas por los colectivos de estudiantes.

Palabras Clave: Estudiantes; Congreso; Grado en Medicina; Educación Médica; Competencias clínicas; Innovación; Docencia; Activismo; Universidad de Alcalá.

Abstract: The XII Congress of Medical Education organized by the State Council of Medical Students (CEEM) with headquarters at the University of Alcalá is the most important annual meeting for medical students nationwide. This event aims to expand the training in scientific and social aspects of future health professionals. In this study the usefulness and quality of learning based on congresses is analyzed through two surveys that were sent to the medical students before and after the congress. For the statistical analysis, we used a proportion comparison test. Our results show the effectiveness of the Congress as an innovative learning tool in the Degree in Medicine. For this reason, it is considered appropriate to keep promoting the activities organized by the student collectives.

Key words: Student; Congress; Medicine degree; Medical Education; Clinic Competence; Innovation; Teaching; Activism; University of Alcala.

\section{Introducción}

El XII Congreso de Educación Médica (CEM) celebrado en Alcalá de Henares del 13 al 16 de marzo de 2019 ha sido el encuentro anual más importante para estudiantes de medicina a nivel nacional reuniendo cerca de 700 participantes [1,2]. El evento, organizado de forma conjunta por el CEEM (Congreso Estatal de Estudiantes de Medicina) y el Comité Organizador (CO) compuesto por estudiantes y representantes de la Universidad de Alcalá (UAH), ha tenido como principales objetivos alcanzar la excelencia docente y ampliar la formación del estudiantado como futuros profesionales de la salud.

El evento surge en el marco reivindicativo de nuevas formas de aprendizaje donde el estudiante es el protagonista de su propia formación y se aplican nuevas tecnologías que permiten poner en práctica los conocimientos adquiridos durante el grado de Medicina. Para ello el Comité Organizador ha seguido un estricto proceso de selección del contenido científico en forma de conferencias, mesas 
redondas y talleres teniendo en cuenta aquellos aspectos que quedan al margen en el Grado en Medicina, como pueden ser la comunicación con el paciente, feminismo, comunidad LGTBI+, emergencias sanitarias, etc.

A nivel económico, al estar organizado por estudiantes, fue fundamental la elaboración de un presupuesto previo viable. Los valores éticos y ecológicos fueron las variables empleadas para la búsqueda de patrocinadores. Sin olvidar la importancia de la enorme participación voluntaria de todos los profesionales sanitarios, instituciones y estudiantes sin la cual el evento no hubiera sido viable.

Dentro de la comunidad médica los congresos están adquiriendo cada vez mayor importancia al ser el punto de reunión de profesionales, a nivel nacional e internacional, para tratar temas de actualidad y avances científicos. ¿Y por qué no para estudiantes? [3-5]

Una vez finalizado el evento, el comité organizador hizo balance de todo el trabajo realizado y analizó la repercusión de este tipo de trabajos. ¿Es eficaz el aprendizaje basado en congresos para el estudiantado de medicina?

El objetivo principal de este trabajo es valorar la utilidad de los eventos organizados por y para estudiantes de cara a la formación en el Grado en Medicina. Secundariamente se persigue evaluar la calidad y el grado de cumplimiento de los objetivos planteados inicialmente en la organización del XII CEM.

\section{Material y Métodos}

La población de estudio para valorar la eficacia en el aprendizaje basado en congresos fue el estudiantado de medicina a nivel nacional y se realizó mediante dos formularios de Google Forms enviados en dos fases distintas:

- El formulario de la primera fase fue enviado vía email y vía Whatsapp al estudiantado a través de las delegaciones de estudiantes de medicina de todo el país. Se recogió la opinión días previos a la celebración del XII CEM tanto de futuros congresistas como de estudiantes que no asistieron.

- El formulario de la segunda fase fue enviado vía email y vía Whatsapp únicamente a los congresistas del XII CEM.

Se realizaron las mismas preguntas multi-respuesta en ambos formularios de manera que se pudiera realizar la comparación entre ambos grupos. Las variables recogidas se agruparon en tres temáticas principales:

- Variables sociodemográficas: edad, sexo, facultad y año académico.

- Conocimientos previos en relación con activismo estudiantil: organización de eventos, asociaciones de estudiantes, valoración acerca de la participación estudiantil en la universidad y su repercusión.

- Crítica y valoración una vez finalizado el evento.

Para el análisis estadístico de los resultados se utilizó el programa RStudio y un test de diferencias porcentuales.

Para la valoración del impacto del CEM se evaluó la existencia de diferencias significativas entre las respuestas recibidas en la primera y la segunda encuesta. 


\section{Resultados}

\subsection{Identificación de nuestra población de estudio:}

En todo el documento nos referiremos como antes y después a los grupos de personas que realizaron la encuesta antes o después del congreso, respectivamente.

Antes de empezar, notamos que hay diferentes encuestas que corresponden al mismo email. Al desconocerse el motivo por el cual han realizado varias encuestas optamos por filtrar los valores repetidos y quedarnos sólo con el último enviado. De la primera encuesta se descartaron 8 respuestas, quedando 242 respuestas válidas. De la segunda encuesta se descartaron 2 respuestas, quedando 109 respuestas válidas.

Son analizadas todas las respuestas de ambos test, siendo 76 personas encuestadas quienes realizaron la primera y la segunda encuesta.

\subsection{Variables sociodemográficas}

\subsubsection{Edad}

La distribución de "Edad" en los grupos antes y después es muy similar. Observamos dos cambios:

- Hay una persona de 41 años (atípica en la encuesta) que además no aparece en el grupo después.

- La mayor parte de las personas de 18 años que respondieron antes se pierden después.

Excluyendo a los individuos de 18 y 41 años las distribuciones son muy similares.

\subsubsection{Sexo}

Respecto a la variable sexo hay 1 individuo con valor "No binario" y 2 "Prefiero no decirlo". La proporción de hombres y mujeres es similar en ambos grupos.

\subsubsection{Facultad}

Naturalmente, la mayor parte de individuos que respondieron la encuesta son de la UAH. No obstante, la proporción de respuestas de la UAH disminuye considerablemente (respecto al resto de universidades) de antes a después.

\subsubsection{Curso académico}

El curso académico es una variable muy relacionada con la edad. Como era de esperar tras ver la variable "Edad", el único grupo que disminuye considerablemente en proporción es el de 1‥ El resto mantiene una proporción similar. Destacar que los estudiantes de $2^{\mathrm{o}}$ curso han respondido en un número bastante bueno en la segunda encuesta.

\subsubsection{Personas que hicieron ambas encuestas}

En total tenemos 242 individuos antes y 107 después. Nos servimos del email de contacto para detectar cuántos individuos han respondido ambas encuestas. Con ánimo de evitar cualquier sesgo y al tener una muestra suficientemente grande de personas decidimos analizar las respuestas solo de aquellas personas que hayan realizado ambos test (antes y después). 


\section{3. Análisis de respuestas:}

En esta sección analizaremos pregunta a pregunta la proporción de personas que han elegido cada respuesta por cada grupo antes y después.

\subsubsection{Si conoces el CEM ¿a través de qué vía? (Test 1 Pregunta nº 2)}

La mayoría de las personas encuestadas (60,4\%) afirman conocer el Congreso de Educación Médica organizado por el CEEM a través de la delegación de alumno/as de medicina de su facultad y a través de compañero/as de clase. Siendo estos resultados mucho más mayoritarios frente al conocimiento de este evento a través de internet y redes sociales (Figura 1).

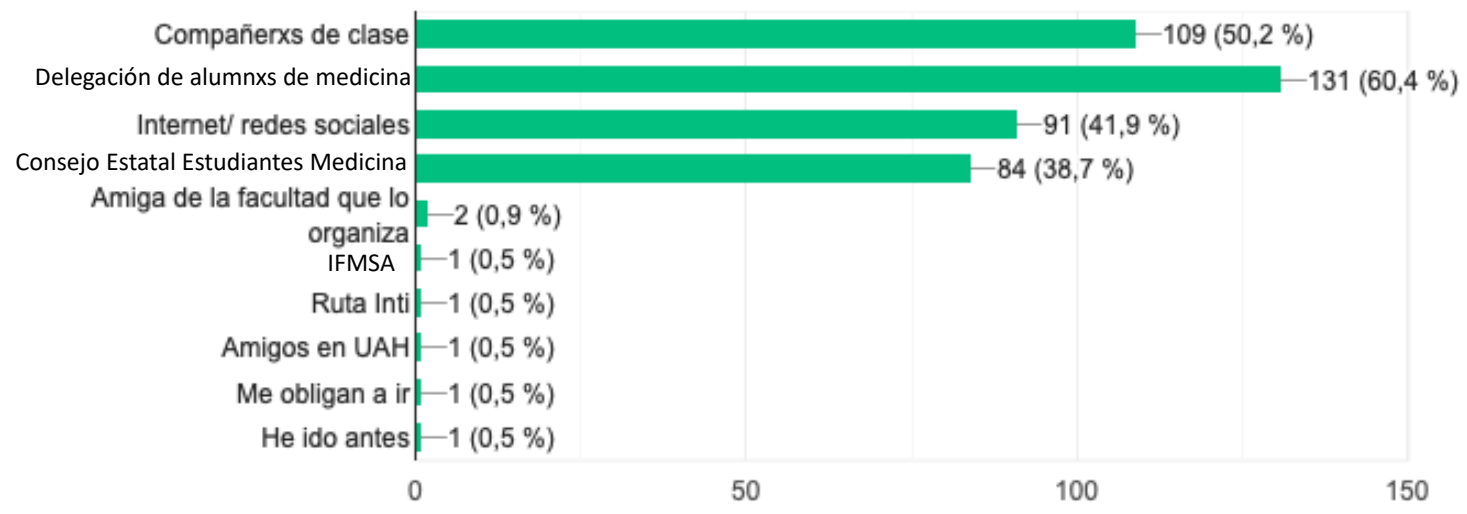

Figura 1. Respuestas de las personas encuestadas sobre la vía de información por la cual han conocido al XII Congreso de Educación Médica del CEEM.

\subsection{2. ¿Por qué te interesaría asistir al XII CEM? (Test 1 Pregunta 4)}

La mayoría respondieron que se debía a querer ampliar su formación (71,6\%). En segundo lugar de frecuencia respondieron que se debía a "porque no han asistido previamente a ningún evento organizado por estudiantes" (49,2\%). Sin embargo, entre las personas que no acudirían al XII CEM respondieron que las razones por las que no acudían se debía "al miedo a perder sus prácticas" $(4,4 \%)$, "no querer perder clase en la facultad" $(8 \%)$ y "por tener exámenes en esas fechas"( $3,6 \%)$.

3.3.3. ¿Consideras que te han resultado útiles en tu formación? (Test 1 Pregunta no 7/ Test 2 Pregunta no 5)

Para analizar esta pregunta nos quedamos solo con estudiantes que antes y después hayan ido a algún congreso. Hay varios estudiantes que nunca han asistido a un congreso por lo que su respuesta antes es "No sabe/No Contesta" (NS/NC) que no se puede contrastar con su opinión después. De esta manera los individuos con los que componemos esta estadística nos sirven para medir cómo ha cambiado el XII CEM su opinión.

Tabla I. Tabla respuestas formulario fase 1 y fase 2 sobre opinión utilidad en la formación$$
\text { despues }
$$$$
\text { Si Tal vez }
$$ \\ antes \\ $\begin{array}{lll}\text { Si } & 34 & 1\end{array}$ \\ Tal vez $28 \quad 0$}

La proporción de "Sí" antes es del 55.5\% y después es de 98.4\%, siendo esta una diferencia estadísticamente significativa con un p-valor de 3.741e-08 (<0.05) asociado. 
3.3.4. ¿Sabes cómo son organizados y por qué colectivos? (Test 1 Pregunta no 8/ Test 2 Pregunta ํㅡㄴ)

A esta pregunta, las personas encuestadas podían responder "Sí", "No" o "No estoy seguro/a, pero me hago una idea". Contrastaremos las proporciones de "Sí" y de "Sí"+ "No estoy seguro/a, pero me hago una idea" antes y después. La proporción de personas que respondieron "Si" es de un 35.5\% antes y un $60.5 \%$ después, siendo esta una diferencia estadísticamente significativa con un p-valor de $0.003475(<0.05)$ asociado.

Por otro lado, la proporción de personas que respondieron "Sí" o "No estoy del todo seguro/a, pero me hago una idea" es de un $86.8 \%$ antes y un $97.4 \%$ después, siendo esta también una diferencia estadísticamente significativa con un p-valor de $0.03524(<0.05)$ asociado.

3.3.5. Si se pudiera organizar desde tu facultad ¿te gustaría participar en la elaboración del mismo? (diseño, colaboración, formación...) (Test 1 Pregunta n⿳o 9/ Test 2 Pregunta no 7)

Tabla II. Tabla respuestas formulario fase 1 y fase 2 sobre interés en la realización y organización de eventos formativos

\begin{tabular}{crrr} 
& \multicolumn{2}{c}{ despues } \\
\cline { 2 - 4 } antes & \multicolumn{2}{c}{ No Tal vez } \\
No & 2 & 7 & 5 \\
Si & 3 & 18 & 16 \\
Tal vez & 2 & 12 & 11
\end{tabular}

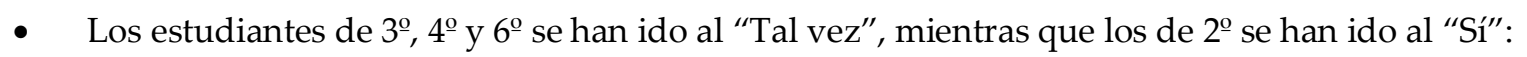

Tabla III. Evolución de proporciones de respuestas en fase $1 \mathrm{y}$ fase 2 por cursos.

Evolución de proporciones de antes a despues por categorías:

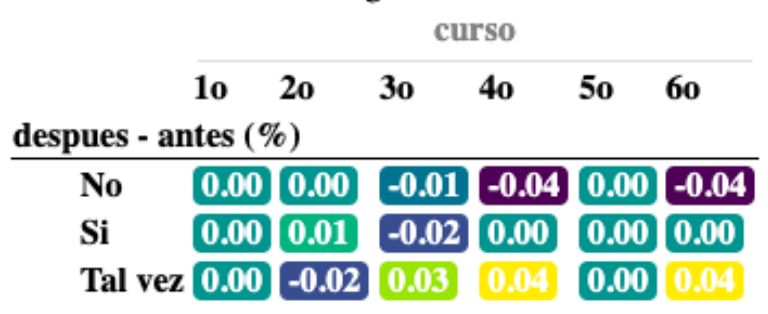

- Los estudiantes hombres se han ido mayoritariamente al "si", mientras que las mujeres estudiantes se han ido al "tal vez":

Tabla IV. Evolución de proporciones de respuestas en fase 1 y fase 2 por sexo.

Evolución de proporciones de antes a despues por categorías:

sexo

Hombre Mujer Prefiero no decirlo

despues - antes (\%)

No $\quad \begin{array}{ccc}-0.05 & -0.04 & 0.00\end{array}$

$\begin{array}{llll}\mathrm{Si} & \mathbf{0 . 0 4} & \mathbf{- 0 . 0 4} & \mathbf{0 . 0 0}\end{array}$

Tal vez $0 . 0 1 \quad 0 . 0 8 \longdiv { 0 . 0 0 }$ 
- A excepción de los estudiantes de 19 años, hay una tendencia clara en el cambio del "no" en "tal vez":

Tabla V. Evolución de proporciones de respuestas en fase $1 \mathrm{y}$ fase 2 por edad. Evolución de proporciones de antes a despues por categorías:

\begin{tabular}{|c|c|c|c|c|c|c|c|c|c|c|}
\hline spues - an & $\begin{array}{l}19 \\
\text { ntes (\%) }\end{array}$ & 20 & 21 & 22 & 23 & 24 & 25 & 27 & 28 & 31 \\
\hline No & 0.03 & -0.03 & -0.04 & -0.02 & -0.02 & -0.02 & -0.02 & 0.00 & 0.00 & 0.00 \\
\hline $\mathbf{S i}$ & -0.01 & 0.02 & -0.01 & 0.00 & 0.00 & 0.00 & 0.00 & 0.00 & 0.00 & 0.00 \\
\hline Tal vez & -0.04 & 0.04 & 0.02 & 0.03 & 0.01 & 0.01 & 0.01 & 0.00 & 0.00 & 0.00 \\
\hline
\end{tabular}

La proporción de personas que "Sí" participarían es la misma antes y después (48.7\%) sin diferencias estadísticamente significativas.

3.3.6. ¿Participas en alguna asociación de colectivos de estudiantes? (Test 1 Pregunta $n^{\circ}$ 12/ Test 2 Pregunta no 10 )

Se observó que 22 personas encuestadas después del congreso participan en asociaciones estudiantiles, mientras que 52 personas no (Tabla VI).

Tabla VI. Tabla respuestas formulario fase 1 y fase 2 sobre participación y activismo en colectivos de estudiantes

\begin{tabular}{ccr} 
& \multicolumn{3}{c}{ despues } \\
\cline { 3 - 3 } antes & No & Si \\
No & 38 & 14 \\
Si & 16 & 8
\end{tabular}

3.3.7. En relación con la pregunta anterior ¿por qué participas o no en alguna asociación de colectivos de estudiantes? (Test 1 Pregunta no 13 )

Las respuestas a esta preguntan se enmarcan en una participación debida a que valoran positivamente la importancia de su repercusión $(33,2 \%)$, complementa su formación personal (36,4\%), complementa su formación académica $(34,4 \%)$. Un $44,8 \%$ de las personas encuestadas consideran no tener tiempo para poder involucrarse.

3.3.8. ¿Cómo valoras que sean lo/as estudiantes quienes organicen este tipo de actividades para estudiantes? (Test 1 Pregunta no 15 / Test 2 Pregunta $\mathrm{n}^{\circ}$ 13)

Tabla VI. Tabla respuestas formulario fase 1 y fase 2 sobre valoración sobre quien organiza eventos formativos.

$$
\text { despues }
$$

Indiferente, da igual quien lo organice Muy positivo Positivo

antes

$\begin{array}{lrrr}\text { Indiferente, da igual quien lo organice } & 0 & 3 & 1 \\ \text { Muy positivo } & 2 & 51 & 7 \\ \text { Positivo } & 0 & 7 & 5\end{array}$

La proporción de personas que han respondido "Positivo" o "Muy Positivo" fue de un 94.7\% antes y de un $97.4 \%$ después. La diferencia no es estadísticamente significativa, por lo que el CEM no parece tener una influencia en esta respuesta. 
3.3.9. ¿Crees que tiene más, menos o igual efecto que si no lo organizaran estudiantes? Por ejemplo, industrias farmacéuticas, profesorado, sociedades científicas, etc. (Test 1 Pregunta no 16/ Test 2 Pregunta no 14)

Tabla VII. Tabla respuestas formulario fase 1 y fase 2 sobre si el efecto/impacto depende de quién organiza un evento formativo para el estudiantado.

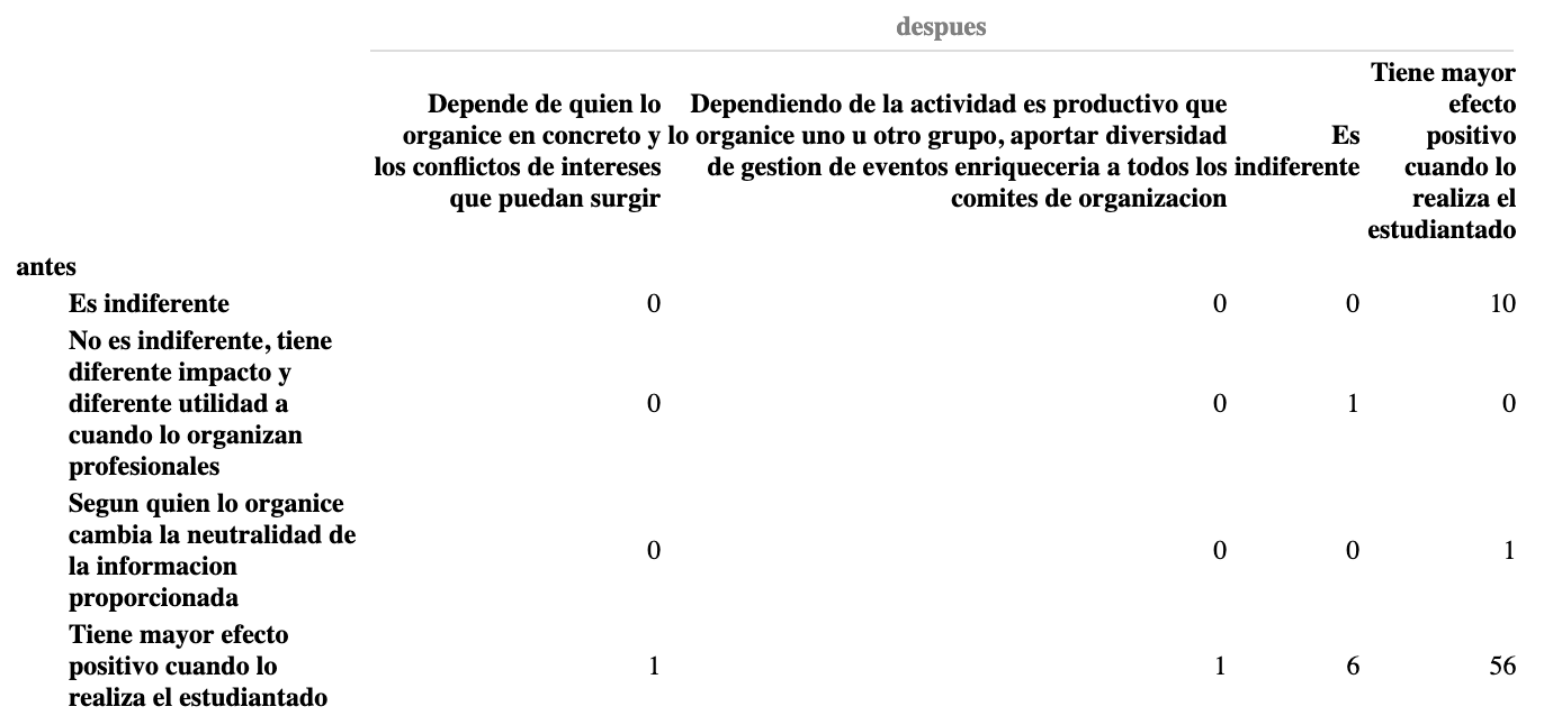

Al igual que en la pregunta anterior, la proporción de personas que opinan que "Tiene mayor efecto positivo cuando lo realiza el estudiantado", no cambia significativamente de antes $(84.2 \%)$ a después $(88.2 \%)$, pero el porcentaje de opiniones en favor a que estas iniciativas sean organizadas por estudiantes vuelve a ser muy superior al de cualquier otra opinión.

3.3.10. Si pudieras tomar la decisión de completar o ampliar tu formación ¿lo harías? Por ejemplo, la organización/ participación en talleres, charlas, conferencias... (Test 1 Pregunta no 19/ Test 2 Pregunta no 17)

Tabla VIII. Tabla respuestas formulario fase 1 y fase 2 sobre participación e involucración personal.

\section{despues}

\section{Si Tal vez}

antes

$\begin{array}{lrr}\text { Si } & 57 & 8 \\ \text { Tal vez } & 10 & 1\end{array}$

La proporción de "Sí" pasa de un $85.5 \%$ a un $88.2 \%$ lo que no constituye una diferencia estadísticamente significativa. Aunque nuevamente, la opinión positiva es la más popular con diferencia. 
3.3.11. ¿Qué temas consideras que son necesarios impartir como formación al estudiantado de medicina y actualmente están ausentes? (Test 1 Pregunta ํำ 24)

Existen competencias que las personas encuestadas destacaron como necesarias entre el estudiantado de medicina, pero insuficientes en la planificación de los estudios en el Grado en Medicina como destrezas prácticas clínicas y técnicas de comunicación $(66,4 \%)$, emergencias sanitarias $(64,4 \%)$, simulación y manejo clínico mediante casos prácticos $(56,4 \%)$, igualdad efectiva de género $(52,4 \%)$, atención primaria $(48,8 \%)$, cuidados paliativos $(44,8 \%)$, conocimientos básicos de medicina general (42,4\%), bioética (39,2\%) y salud pública y medicina preventiva (29,6\%)(Figura 2).

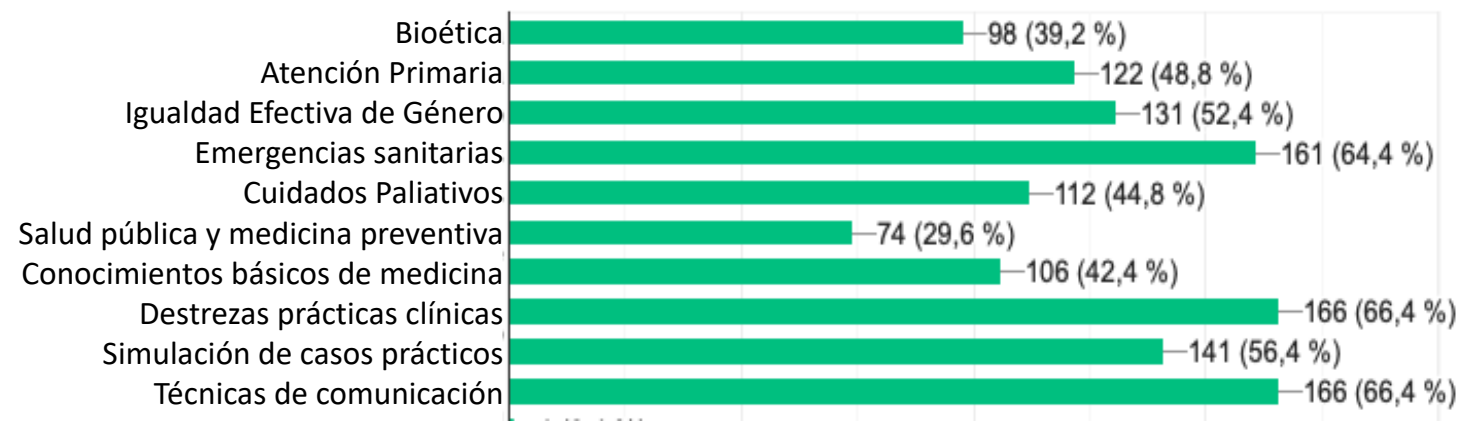

Figura 2 Respuestas en test fase 1 de las personas encuestadas a la pregunta "¿Qué temas consideras que son necesarios impartir como formación al estudiantado de medicina y actualmente están ausentes? (marca todas las que consideres)".

\subsubsection{2. ¿Volverías a asistir a un evento de estas características? (Test 2 Pregunta no 23)}

Dentro de las respuestas en la segunda fase, el 99,1\% de las personas encuestadas afirman que volverían a asistir a un evento para estudiantes de estas características.

\subsubsection{3. ¿Lo recomendarías a tus compañero/as de clase/facultad? (Test 2 Pregunta oํㄹ)}

Siendo el $100 \%$ de las personas encuestadas quienes afirman que lo recomendarían a sus compañero/as de clase y facultad.

3.3.14. En relación con los contenidos ¿en qué te has basado para elegirlos cuando te inscribiste? (Test 2 Pregunta no 25)

Según las respuestas obtenidas en el test de la segunda fase donde se preguntaba por las razones por las cuales los congresistas elegían la temática de sus contenidos encontramos que el $84,4 \%$ de ellos lo eligen ya que les llama la atención, el $67 \%$ lo eligieron por el contenido práctico, el 59,6\% por el crecimiento personal, el 51,6\% por cultura general sanitaria, el 50,5\% por estar relacionados con la especialidad que les gusta, el $36,7 \%$ lo ha elegido por el contenido reflexivo-personal y el $31,2 \%$ lo ha elegido por no impartirse en su facultad (Figura 3).

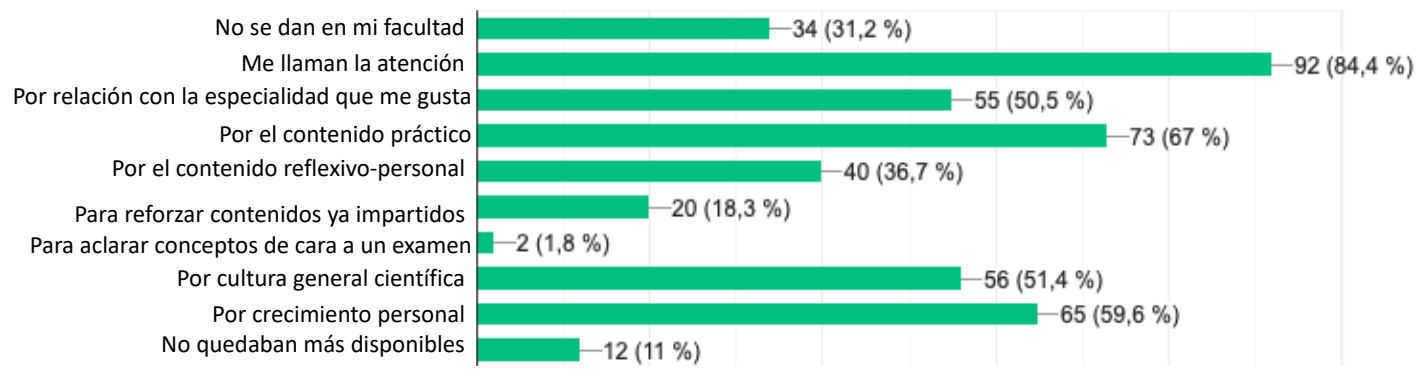

Figura 3 Respuestas en test fase 2 de las personas encuestadas a la pregunta "En relación con los contenidos ¿en qué te has basado para elegirlos cuando te inscribiste? (marca todas las que consideres)". 
3.3.15. ¿Crees que has acertado con tus elecciones? (Test 2 Pregunta no ${ }^{26}$ )

El 94,5\% de las personas encuestadas considera que ha acertado con sus elecciones.

3.3.16. Asistir al XII CEM, ¿te ha aportado algo más a parte del conocimiento en sí? (Test 2 Pregunta nº 28)

Encontramos un 70,6\% que considera que el XII CEM le ha aportado mucho más a parte de conocimiento en sí y un 29,4\% que considera que algo más (Figura 4).

\section{9 respuestas}

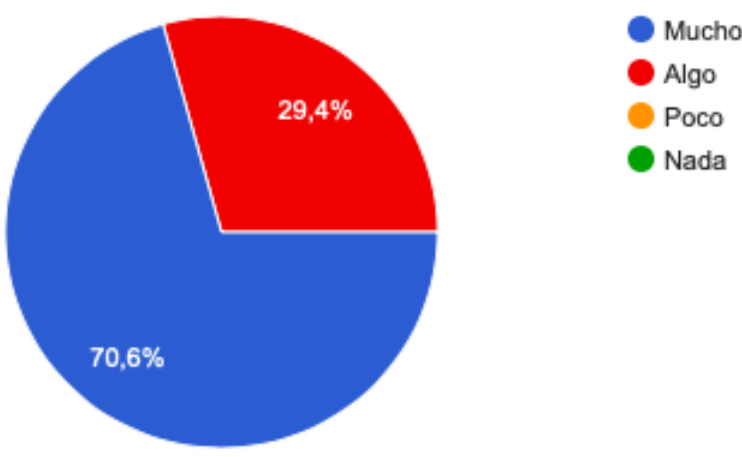

Figura 4 Respuestas en test fase 2 de las personas encuestadas a la pregunta "Asistir al XII CEM, ¿te ha aportado algo más a parte del conocimiento en sí?".

3.3.17. ¿La información que has recibido previamente al congreso se ha ajustado a lo que has vivido? (Test 2 Pregunta no 29)

\section{9 respuestas}

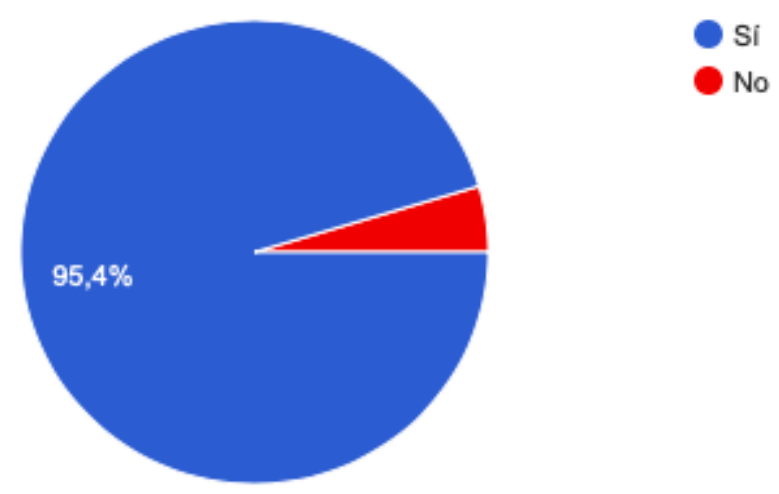

Figura 5 Respuestas en test fase 2 de las personas encuestadas a la pregunta "¿La información que has recibido previamente al congreso se ha ajustado a lo que has vivido?". 
El 95,4\% afirmó que la información recibida antes del congreso se ajustó a lo vivido durante el mismo (Figura 5). El 4,6\% de personas encuestadas que respondieron que la información recibida previamente al congreso no se ha ajustado a lo que ha vivido justificaron las siguientes situaciones:

- "Ha sido mucho mejor de lo que esperaba".

- "Me lo esperaba peor y ha sido mucho mejor".

- “Un poco más de organización”.

- "Se debería dar una pequeña definición del contenido de talleres, hay algunos que tienen un título que puede dar lugar a error o que es tan amplio que puede que lo que se trate no es lo que esperabas. Además, creo que es importante distinguir entre seminarios y talleres, un taller es más práctico que un seminario".

\section{Discusión}

La selección de la población estuvo limitada por el interés personal del estudiantado en contestar ambos test. De cara a futuros estudios, es recomendable ampliar la muestra poblacional para garantizar una mayor evidencia científica.

Dentro de las variables sociodemográficas, la mayor parte de las personas de 18 años que respondieron antes se pierden después. Probablemente porque no irían al congreso o no estén todavía lo suficientemente involucrados con este tipo de iniciativas (estudiantes de primer año de grado). Los estudiantes de $2^{\circ}$ curso respondieron en un número bastante bueno en la segunda encuesta.

Con respecto a la Facultad de origen de los encuestados, mayor parte de los que respondieron la primera encuesta fueron de la UAH. No obstante, la proporción de respuestas de la UAH disminuye considerablemente (respecto al resto de universidades) de antes a después. El amplio porcentaje de respuestas en el primer test podría explicarse por ser la facultad en la que se realizaba el congreso y el estudio y la baja respuesta de este grupo en el segundo formulario podría derivarse de que muchos estudiantes formaron parte del equipo de personas voluntarias.

Nuestro estudio muestra que las vías más eficaces para la divulgación de información entre lo/as estudiantes de Medicina fueron la llevada a cabo por las diferentes delegaciones de alumno/as y la comunicación entre compañero/as. Esto refleja la importancia de la labor de estas asociaciones. Concretamente, gracias al trabajo de comunicación interna por parte del CEEM se facilita la difusión a todas las delegaciones de estudiantes de medicina. Además, según nuestros resultados, acudir al CEM es estadísticamente significativo para dar a conocer a la asociación estudiantil organizadora, al CEEM en este caso.

Aunque minoritario, el "miedo a perder prácticas, o clase en la facultad" no debería ser un motivo por el que dejar de acudir a este tipo de eventos, pues enriquecen y complementan la formación como futuros profesionales de la salud. Por ello se anima a las facultades y profesorado a facilitar la asistencia a estas actividades. Por ejemplo, reconocerlo como créditos de libre elección, justificar la falta de asistencia y facilitar el transporte a la ciudad donde se celebre el evento.

Con relación a la utilidad del congreso en la formación, la diferencia estadísticamente significativa observada entre el porcentaje de personas que consideraban que sí era útil antes de acudir y el porcentaje de participantes que consideró que sí fue útil parece apoyar el hecho de el aprendizaje basado en congresos es válida dentro de la formación en el Grado en Medicina.

Tras finalizar el Congreso, se preguntó por el interés de organizar este tipo de evento en su facultad y resultó llamativo que el curso en el que se obtuvo un mayor número de respuestas positivas fue $2^{2}$. Esto podría explicarse porque en este curso los estudiantes ya conocen algunas asociaciones dentro de la universidad y aun no tienen tanta carga académica clínica. Parece que conforme se acerca el final de la carrera y con ello el estudio del examen MIR se pierden algunas perspectivas holísticas de la formación como futuro profesional de la salud. Además, el porcentaje de asertividad en las respuestas "Sí me animo a organizar este tipo de eventos" fue mayor en los hombres que en las mujeres ("Tal vez me animaría"), lo que podría estar relacionado con estigmas sociales que perpetúan en nuestros días y que hacen que la mujer se encuentre en una posición de inferioridad para el liderazgo. El ambiente feminista que se ha construido durante el congreso no ha sido suficiente, por 
lo que es necesario seguir fortaleciendo y empoderando a las mujeres para que se sientan capaces de poder ocupar puestos de organización y dirección.

La ausencia de cambio en el interés para participar en asociaciones de estudiantes podría deberse a que el congreso se celebró en mitad del curso académico, dificultando los cambios en la organización semanal de actividades de los congresistas. También podría ser por la diversidad de intereses personales entre el total de congresistas y la falta de tiempo libre que manifiestan las personas encuestadas. Quizá, es posible que, si este tipo de eventos se celebraran al comienzo de curso, sí anime al estudiante a participar activamente.

La práctica totalidad de las personas encuestadas consideran "Positivo" o "Muy positivo" que sean los propios estudiantes los que organicen este tipo de actividades para estudiantes. Además, muchas de estas personas argumentan que su respuesta en base a la ausencia de conflictos de intereses que sí pudieran estar presentes cuando son organizados por sociedades o industria farmacéutica.

En cuanto a la valoración de las temáticas consideradas por el estudiantado como necesarias en su formación, pero ausentes/deficientes en el Grado las principales que destacan fueron: contenido práctico, técnicas de comunicación e igualdad efectiva de género. Estas temáticas fueron las que tuvieron más peso en el congreso, coincidiendo, además, muchas de ellas con las líneas estratégicas de trabajo anuales del CEEM (salud pública, atención primaria, bioética, etc.) [6].

En definitiva, el Congreso ha permitido por una parte ampliar y consolidar las habilidades clínicas a través de talleres prácticos y fomentar el espíritu crítico del estudiante; y por otro lado promover la Universidad como un lugar de punto de encuentro de actividades científico-sanitarias, así como culturales, artísticas, literarias y reivindicativas [1,2].

\section{Conclusiones}

- Como muestra nuestro estudio, es útil para la formación en el Grado en Medicina el aprendizaje basado en congresos.

- Es posible conseguir mediante una formación de calidad y distintas metodologías docentes, que el estudiantado de grados de ciencias de la salud desarrolle una actitud activa, participativa, madura, crítica y constructiva, que le permita convertirse en verdaderos protagonistas de su formación.

- Es imprescindible el trabajo realizado por las delegaciones de estudiantes de medicina de las distintas facultades del país para dar a conocer los eventos realizados por colectivos de estudiantes, como el CEEM.

- Es necesaria la implicación del profesorado y la facultad para facilitar la asistencia a los eventos formativos organizados por asociaciones y colectivos de estudiantes, como el CEEM.

- Ceder el protagonismo al estudiante de medicina en la elección de los contenidos (mesas redondas, talleres y conferencias) facilita cumplir con sus preferencias personales o carencias formativas en función al curso académico en el que se encuentra.

- Las expectativas e ideas preconcebidas previas al evento son en general muy positivas; manteniéndose positivas una vez finalizado el evento.

- El Congreso de Educación Médica nos ofrece un programa de elevado carácter científico y humano, consiguiendo superarse y mejorar cada año, teniendo cada vez más impacto a nivel nacional. 
Agradecimientos: En primer lugar, agradecemos al Decanato de la Facultad de Medicina y Ciencias de la Salud, especialmente al Dr. Manuel Rodríguez Zapata, Dra. Lourdes Lledó García y Dra. Consuelo Giménez Pardo la ayuda, el esfuerzo y la confianza depositada en el comité organizador del XII CEM. Agradecemos a la Universidad de Alcalá el apoyo para la realización del evento sin la cual no hubiera sido posible. Agradecemos a todas las personas que de una manera directa participaron en el XII CEM como ponentes, voluntarios, organizadores, etc. Gracias al CEEM por la oportunidad de ser la sede de la XII edición del Congreso de Educación Médica y su apoyo durante todo el proceso. Agradecemos a Miguel Ángel Fernández Guerrero la ayuda recibida para el análisis estadístico del estudio. Dedicamos una mención especial a Alba, Alba M., Alejandro, Amanda, Amalio, Blanca, Candela, Coral, Daniel, David, Eloísa, Fernando, Lucía, Paula, Raquel, Rosa, Sandra y Teresa por todo su trabajo, ilusión y perseverancia desde que se empezó a esbozar este proyecto. Por último, agradecemos al Equipo Editorial de la presente revista electrónica por haberse puesto en contacto con nosotras y dar altavoz a nuestro trabajo de preparación y difusión del congreso.

Contribución de los autores: Celia Estrada Costas y Paula Rubio García han concebido y diseñado el estudio; Celia Estrada Costas y Paula Rubio García realizaron la revisión bibliográfica histórica; Celia Estrada Costas y Paula Rubio García escribieron el artículo.

Conflictos de Intereses: Los autores no declaran conflicto de intereses. Los patrocinadores fundadores no tenían ningún papel en el diseño del estudio; en la colección, análisis o interpretación de los datos; en la escritura del manuscrito y en la decisión de publicar los resultados.

\section{Abreviaturas}

Las siguientes abreviaturas son usadas en este manuscrito:

CEM: Congreso Educación Médica.

CEEM: Consejo Estatal de Estudiantes de Medicina.

CO: Comité Organizador.

NS/NC: No sabe/No contesta

UAH: Universidad de Alcalá.

XII CEM: XII Congreso de Educación Médica

\section{Referencias Bibliográficas}

1. Estrada Costas CM, Rubio García P. Congreso de Educación Médica hecho por y para estudiantes. RIECS, UAH [Internet]. 2019;2-6. Disponible en: https://riecs.es/index.php/riecs/article/view/111

2. https://www.youtube.com/watch?v=mMbv6N8 p8s

3. Más de 600 estudiantes de Medicina de toda España se reúnen en la UAH para celebrar el XII Congreso de Educación Médica [Internet]. [cited 2019 May 28]. Disponible en: http://portalcomunicacion.uah.es/salaprensa/notas-prensa/mas-de-600-estudiantes-de-medicina-de-toda-espana-se-reunen-en-la-uah-paracelebrar-el-xii-congreso-de-educacion-medica.html

4. Más de 600 estudiantes de Medicina de toda España se reúnen en la UAH para celebrar el XII Congreso de Educación Médica [Internet]. [cited 2019 May 28]. Disponible en: http://portalcomunicacion.uah.es/salaprensa/notas-prensa/mas-de-600-estudiantes-de-medicina-de-toda-espana-se-reunen-en-la-uah-paracelebrar-el-xii-congreso-de-educacion-medica.html

5. Comienza el XII Congreso de Educación Médica en la Universidad de Alcalá [Internet]. [cited 2019 May 28]. Disponible en: https://www.ceem.org.es/2019/2019/03/14/comienza-el-xii-congreso-de-educacionmedica-en-la-universidad-de-alcala/

6. Consejo Estatal Estudiantes de Medicina CEEM. ESTATUTOS DEL CONSEJO ESTATAL DE ESTUDIANTES DE MEDICINA. 2002; Available from: http://www.ceem.org.es/web/foro/ 